\title{
Thermodynamical versus log-Poisson distribution in turbulence
}

\author{
Guowei He ${ }^{\mathrm{a}, \mathrm{b}}$, B. Dubrulle ${ }^{\mathrm{c}, \mathrm{d}}, \mathrm{F}$. Graner ${ }^{\mathrm{e}}$ \\ a Theoretical Division and Complex Systems, Los Alamos National Laboratory, Los Alamos, NM 87545, USA \\ ${ }^{\mathrm{b}}$ LNM, Institute of Mechanics, Chinese Academy of Sciences, Beijing, China \\ ' CNRS, URA 2052, CEA/DSM/DAPNIA/Service d'Astrophysique, CE Saclay, F-91191 Gif sur Yvette, France \\ ¿ CNRS, URA 285, Observatoire Midi-Pyrénées, 14 avenue Belin. F-31400 Toulouse, France \\ e CNRS, UMR 5588, Laboratoire de Spectrométrie Physique, Université Grenoble I, BP 87, F-38402 Saint-Martin d'Hères, France
}

Received I December 1997; revised manuscript received 3 May 1998; accepted for publication 8 June 1998

Communicated by A.R. Bishop

\begin{abstract}
The thermodynamical model of intermittency in fully developed turbulence due to Castaing (B. Castaing, J. Phys. II France 6 (1996) 105) is investigated and compared with the log-Poisson model (Z-S. She, E. Lévêque, Phys. Rev. Lett. 72 (1994) 336). It is shown that the thermodynamical model obeys general scaling laws and corresponds to the degenerate class of scale-invariant statistics. We also find that its structure function shapes have physical behaviors similar to the log-Poisson's one. The only difference between them lies in the convergence of the log-Poisson's structure functions and divergence of the thermodynamical one. As far as the comparison with experiments on intermittency is concerned, they are indifferent. (C) 1998 Published by Elsevier Science B.V.
\end{abstract}

In isotropic turbulence at high Reynolds numbers, for scales in the so-called inertial range, the velocity field $\boldsymbol{u}$ has structure functions $\left|\delta u_{\ell}\right|^{n} \equiv$ $\left\langle|\boldsymbol{u}(x+\ell)-\boldsymbol{u}(x)|^{n}\right\rangle$ which go as power laws of the scale $\ell:\left|\delta u_{\ell}\right|^{n} \sim \ell^{\zeta(n)}$. Moreover, the scaling exponents $\zeta(n)$ appear nearly independent of flow geometry or Reynolds number, at least for $n \leqslant 8$ [1]. A dimensional analysis due to Kolmogorov [2] predicts $\zeta(n)=n / 3$. Present experimental data are not consistent with such a linear shape and this discrepancy is a central question in turbulence [3]. Among the various answers found in the literature, two models have recently attracted special attention.

- She and Lévêque [4], using a hierarchical description of energy transfers, obtain an excellent fit of the data with no adjustable parameter,

$$
\zeta^{s L}(n)=\frac{n}{9}+2\left[1-\left(\frac{2}{3}\right)^{n / 3}\right]
$$

later shown to correspond to a log-Poisson distribution for the velocity increments $[5,6]$.

- Castaing [7], with a thermodynamical approach, obtains

$\zeta^{C}(n)=\frac{n}{3} \frac{1+3 T}{1+n T}$,

where the "temperature" $T$ of the turbulent flow is inversely proportional to the Reynolds number. A value of $T=0.03$ is sufficient to provide an excellent fit to the experimental data of e.g. Ref. [1]. This confirms that scaling exponents are not a good way to discriminate between various models, as already emphasized in Refs. [8-10]. What then can we do? 
In the present Letter, we consider the constraints imposed by scale invariance, in the spirit of Nottale and Pocheau [11,12]. As was shown in Ref. [10,13-15], scale symmetry can be used to compute the possible scale dependence of the structure functions, i.e. the shape of scaling exponents, and handle infinitely divisible processes. The starting point of these computations is the observation that both universality and nonuniversality coexist in turbulent flows. Some scaling properties are universal; for instance, relative scaling exponents $\zeta(n) / \zeta(3)$ are independent of the geometry (cylinder, jet, grid) [1], of the dissipation [16] or of the type of turbulence (hydrodynamical, convection, magneto-hydrodynamical) [17]. Yet, the most intermittent structures, representing large but rare velocity fluctuations, are not universal; they look like filaments or worm in numerical 3D turbulence [18], in helium or grid generated turbulence $[19,20]$; while they are vorticity rings in jet turbulence [20], and thermal plumes in convection or magnetic sheets in magneto-hydrodynamics [21].

This observation led Dubrulle and Graner $[13,10]$ to separate into two parts the velocity structure functions, and their scaling exponents in the inertial range,

$$
\begin{aligned}
& \left\langle\left|\delta u_{\ell}\right|^{n}\right\rangle=\left(\delta u_{\ell}^{\infty}\right)^{n}\left\langle\left|\frac{\delta u_{\ell}}{\delta u_{\ell}^{\infty}}\right|^{n}\right\rangle, \\
& \zeta(n)=n \Delta+\delta \zeta(n) .
\end{aligned}
$$

- The first part, non-universal, is the maximal value of intermittent velocity increments, defined $[4,5,13,10]$ as $\delta u_{\ell}^{\infty} \equiv \lim _{n \rightarrow \infty}\left\langle\left|\delta u_{\ell}\right|^{n+1}\right\rangle /$ $\left\langle\left|\delta u_{\ell}\right|^{n}\right\rangle$. Its scaling exponent in the inertial range is $\Delta$. This non-universal part should be computed according to a given geometry, dissipation or finite size effects [22,23]. For instance, in 3D homogeneous, isotropic, hydrodynamical turbulence, it can be computed using the Kolmogorov 4/5 law directly derived from the Navier-Stokes equation [15].

- The (potentially) universal part $\delta u_{\ell} / \delta u_{\ell}^{\infty}$ is now a dimensionless velocity increment. Its contribution is the intermittency function $\delta \zeta(n)$. The idea is that it should only depend on a "universal" mechanism, common to all configurations whatever the experimental conditions, Reynolds number, turbulence production, turbulence type. One possibility is that they share a symmetry: here the scale invariance. Since the intermittent structures have been renormalized out, they are no longer influent except via the limit $\lim _{n \rightarrow \infty}\left\langle\left(\delta u_{\ell} / \delta u_{\ell}^{\infty}\right)^{n}\right\rangle$. This limit is just the probability to find a velocity increment of amplitude $\delta u_{\ell}^{\infty}$ at scale $\ell$, since by definition $\delta u_{\ell}^{\infty}$ is the largest velocity increment. For small enough $\ell$, this probability scales like $\ell^{C}$, where $C$ is the codimension of the set of the largest velocity increments. Scale symmetry arguments [13] now classify the possible shapes of the intermittency function according only to the geometry of the intermittent structures. Physically, it then only depends on whether there are zero, one or two types of intermittent structures, each characterized by one codimension.

In turbulence, experiments and numerical simulations suggests that there is only one type of intermittent structures, worms or rings according to the turbulence production. In such a case, there are only two possible shapes for the intermittency function [13]: the log-Poisson statistics, with only one finite codimension $C_{-}$,

$\delta \zeta(n)=C_{-}\left(1-\beta^{n}\right)$,

or the degenerate shape, where both codimensions collapse into the same value $C_{0}$,

$\delta \zeta(n)=\frac{n C_{0} \beta}{C_{0}+(n-1) \beta}$.

Here $\beta$ is a free parameter. Note that the lug-Poissun case is always regular, while the degenerate case presents a divergency at an usually negative value $n=1-C_{0} / \beta$.

Comparing Eqs. (1), (2), (3b), (4), (5), we see that the She and Lévêque model corresponds to the $\log$-Poisson case with $\Delta=1 / 9, C_{-}=2$ and $\beta=$ $(2 / 3)^{1 / 3}$, while the Castaing model corresponds to the degenerate case with $\Delta=0, C_{0}=1+1 / 3 T$ and $\alpha=(1+3 T)(3+3 T)$. They are then strictly physically equivalent from the point of view of scale invariance, as far as scaling exponents are concerned: once again, even with the additional requirement of scale symmetry, scaling exponents only are not sufficient to discriminate between both models.

To go one step further, we thus address the question of the scale dependence of the velocity structure functions in both models. Graner and Dubrulle [14], using an analogy with mechanics, suggested a formal- 
ism to predict such a scale dependence. Consider a family of random fields based on velocity increments $\phi_{n}(\ell)=\left|\delta u_{\ell}\right|\left|\delta u_{\ell} / \delta u_{\ell}^{\infty}\right|^{n}$. Each member of the family is analog to a particle following a trajectory given by the $\log$-coordinates

$X_{n}(T)=\ln \left\langle\frac{\phi_{n}(\ell)}{\mathcal{R}_{\ell}}\right\rangle, \quad T=\ln \left(\frac{\ell}{L}\right)$,

where $\left(\mathcal{R}_{k}, L\right)$ are respectively a reference field and a reference scale. For instance, $L$ may be chosen as the Kolmogorov scale $\eta$. As for $\mathcal{R}_{\ell}$, its choice influences the meaning of the log-coordinate. For example, if $\mathcal{R}_{\ell}$ is chosen as $\phi_{0}$, then the velocity of the $n$th particle $V_{n / \mathcal{R}}=\mathrm{d} X_{n} / \mathrm{d} T$ is the scaling exponent of the $n$th order reduced structure function $\left\langle\left|\delta u_{\ell}\right|^{n} /\left(\delta u_{\ell}^{\infty}\right)^{n}\right\rangle$ and coincides with the intermittency function $\delta \zeta(n)$ in the inertial range. In analogy with electro-magnetism, it can be obtained from Euler-Lagrange and Maxwell equations [14]. The scale symmetry requires that these equations should be scale covariant and, thus, constrains the shapes of their solutions.

Physically, the analog $E$ of the electric ficld is a function characterizing the injection and dissipation processes in a turbulent flows. The response of the particle to such an electric field then describes the response of a turbulent flow to these mechanisms, and thus, the cascade process from large to small scales. There is one situation where this cascade is especially simple: when the electric ficld is $X$-independent, i.e., when the interactions do not depend on the amplitude of the velocity increments. In such a case, $X$ translation symmetry holds, and by Noether theorem, there is a conserved quantity along the scales. This situation can then be seen as a generalization of Kolmogorov's assumption [2] by which the energy dissipation $\nu \int_{|x|<\ell}\left(\partial_{x} u\right)^{2} \mathrm{~d} x$ is conserved along the scales. Kolmogorov's hypothesis leads to a very special selfsimilar shape for the structure function $\left\langle\left(\delta \boldsymbol{u}_{\ell}\right)^{n}\right\rangle \sim$ $\ell^{n / 3}$. In the same way, the $X$-independent situations leads to a special form of similarity for the reduced structure functions.

This formalism, focussing on the dependence with $\ell$, was applied up to now only to the $\log$-Poisson model [15]. Two main results were obtained:

(i) The universal part of the structure functions obeys a general scaling property extending from the injection scale down to the smallest resolvable scales, thus explaining the observation of Ref. [17].

(ii) The same linear approximation accounts for a large variety of turbulent flows, from jet to boundary layer turbulence.

Let us now examine in detail whether this analysis can apply to the degenerate case in order to distinguish between both models. A basic ingredients of the dynamical equations is a similarity factor, which reads in the degenerate case $\Gamma=\left(1-V / C_{0}\right)^{-1}$. This similarity factor enters in the definition of the impulsion of a particle via $\mathcal{P}=M C_{0}\left(\Gamma^{2}-1\right) / 2$. If the $n$th particle $\phi_{n}$ does not interact with the other ones, $\phi_{p}(p \neq n)$, the impulsion can be given by EulerLagrange equations, whose general shape is given in Ref. [14]. This equation only depends on the analog of an electric field $E$, through a coupling constant $\alpha_{n}$, which reads in the degenerate case,

$\frac{\mathrm{d} \Gamma_{n}^{2}}{\mathrm{~d} T}=\alpha_{n} E(X, T)$.

By studying the response of a particle to various electric fields, one can prove by computations similar to those described in Ref. [15] that the coupling constants must go as

$\alpha_{n}=\alpha_{0}\left(1+\frac{\beta n}{C_{0}-\beta}\right)^{2}$,

while the similarity factor at initial condition $T=0$ obeys

$\Gamma_{n}(0)=\Gamma_{0}(0)\left(1+\frac{\beta n}{C_{0}-\beta}\right)$.

Here $\beta$ is a parameter and $\alpha_{0}$ is the coupling constant of the "Oth particle".

Now, we must account the essential hypothesis that $E$ is independent on $X$. It will solve the equation obtained as the ratio of Eq. (7) written twice, for both particles $\phi_{n}$ and $\phi_{p}$. Using relation (8) and the initial condition (9), we can solve the ratio equation to get

$\Gamma_{n}(T)=\frac{C_{0}+(n-1) \beta}{C_{0}+(p-1) \beta} \Gamma_{p}(T)$.

Now, we introduce the relative velocity of the $n$th particle with respect to the $p$ th particle [13],

$V_{n / p}=C_{0}\left(1-\Gamma_{n / p}^{-1}\right)$. 
Recalling that $\Gamma_{n / p}=\Gamma_{n} / \Gamma_{p}$ [13], we substitute (10) into (11) to obtain $V_{n / p}=C_{0} \beta(n-$ $p) /\left(C_{0}+(n-1) \beta\right)$, which is a (scale independent) constant. As a special case, we get

$V_{n / 0}=\frac{\mathrm{d} \ln \left\langle\phi_{n} / \phi_{0}\right\rangle}{\mathrm{d} \tau}=\frac{C_{0} \beta n}{C_{0}+(n-1) \beta}$,

where $\tau$ is the proper time of the 0th particle. I.et us pause a moment to study the physical meaning of such a relation. In a fixed reference frame $\left(\mathcal{R}_{\ell}, T\right)$, the $n$th and the 0th particle are both accelerated by the same force, the electric field $E$. In the accelerated reference frame $\left(\phi_{0}, \tau\right)$ of the 0 th particle, the test particle $n$ moves under the conjugate action of an "inertial" force and an external force due to the same electric field. Thus, both forces cancel exactly: in this accelerated frame, the test particle $n$ behaves as a free particle with a constant velocity, a property translated by Eq. (12).

This simple property translates into a remarkable property for the shape of the reduced structure functions. Indeed, integrating the expression (12) with respect to $\tau$, we find that the reduced structure functions obey

$\ln \left\langle\left|\frac{\delta u_{\ell}}{\delta u_{\ell}^{\infty}}\right|^{n}\right\rangle=\frac{C_{0} \beta n}{C_{0}+(n-1) \beta} \tau(T)+f_{n}$,

where the integration constant $f_{n}$ reflects the statistics at injection scale. Such relation is the general scaling described in Ref. [17]. In fact, when $\tau=T \ln (\ell)$, a situation obtained e.g. in the inertial range, it means that reduced structure functions are self-similar and go as $\ell^{\delta \zeta(n)}$; in general, however, $\tau$ is not linear in $T$ because it depends on the external force $E$ via $\Gamma_{0}$,

$\mathrm{d} \tau=\frac{\mathrm{d} T}{\Gamma_{0}}$,

where $\Gamma_{0}$ follows the Euler-Lagrange equation (7). One observes well-defined scaling exponents only when plotting one reduced structure function with respect to another one (e.g., the $n=3$ one), see Figs. 1 and 2. In such a plot, which eliminates $\tau$, one then measures relative scaling exponents, e.g. $\delta \zeta(n) / \delta \zeta(3)$, which are then independent on nonuniversal quantities lumped in $E$. This provides an explanation of the universality properties observed in turbulent flows.

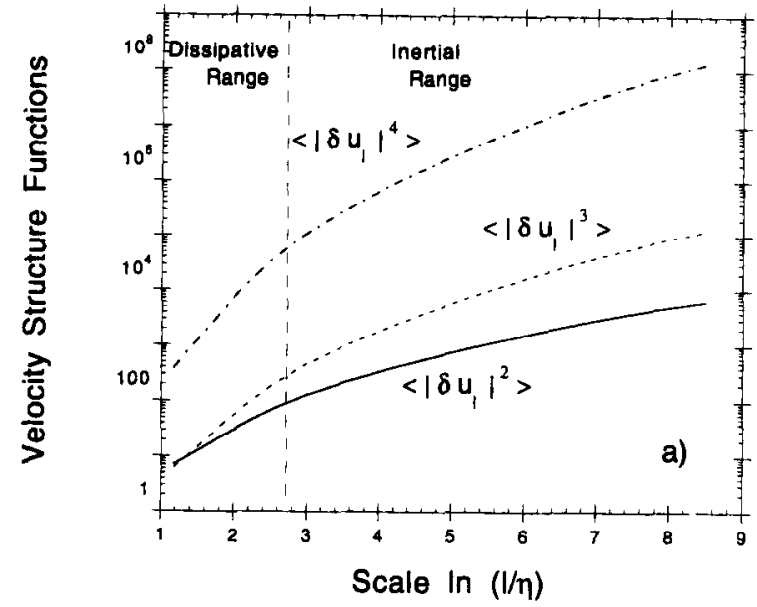

Fig. 1. When plotted against the log-scale $T$, the $n$th order velocity structure functions display a transitional behavior between the anomalous power law $\ell^{\zeta(n)}$ in the inertial range to the regular scaling $\ell^{n}$ in the dissipative range.

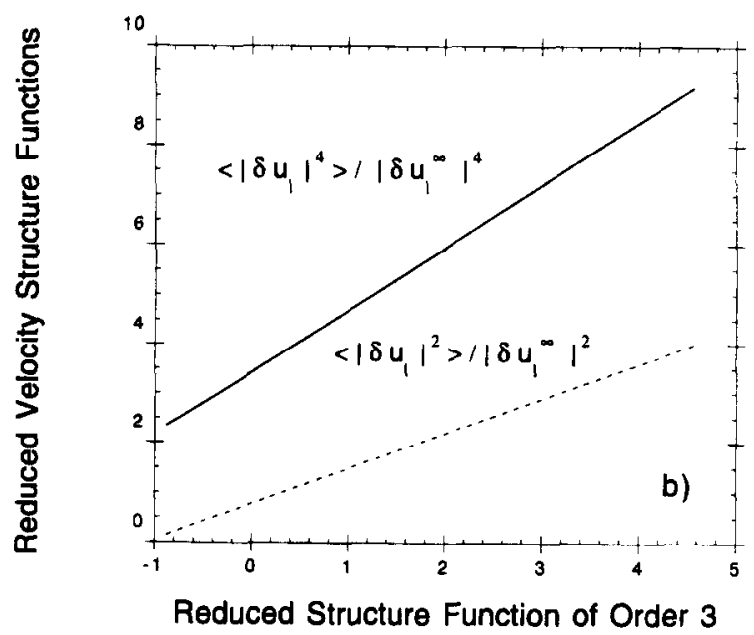

Fig. 2. When the $n$th order reduced structure function is plotted as function of any other one (here the 3rd one). the transition between the inertial range to the dissipative range is erased. All information regarding such a transition is in fact lumped into the function $\tau$ shown in Fig. 3.

To compute explicitely the reduced structure functions, we need the shape of the electric field. The electric field $E$ obeys the Maxwell equation $\partial E / \partial T=-J$ where $J$ is analog to the electric current. We can follow the idea used in the log-Poisson case and consider a linear ("ohmic") approximation $J=\sigma E$, where the proportionality constant is a pseudo-conductivity $\sigma$. This closes the system of equations (7), (14) which 
can be solved using elementary integrations. We can describe all solutions by introducing two characteristic "times" (i.e., log-scales): the injection time $T_{i}$ defined by $\tau\left(T_{i}\right)=0$ and the dissipative time $T_{d}$ by $\Gamma\left(T_{d}\right)=3 / 2$; here the $3 / 2$ is arbitrary, it could be any number larger than one. The solution depends on an integration constant $k=\Gamma_{0}^{2}(0)+\alpha_{0} E_{0} / \sigma$. It yields two different types of solutions:

(1) A scale-symmetric case $k=0$,

$$
\begin{aligned}
& \tau=(4 / 3 \sigma)\left(\mathrm{e}^{\sigma\left(T-T_{d}\right) / 2}-\mathrm{e}^{\sigma\left(T_{i}-T_{d}\right) / 2}\right), \\
& T_{i}=T_{d}+(4 / \sigma) \ln (3 / 2) .
\end{aligned}
$$

This exponential self-similar shape is exactly the same as in the log-Poisson case, except for a factor two in the conductivity. It has been observed in a turbulent wind tunnel experiment [24], and predicted using Lagrangian [25] or normal form [22] approaches. This case is compatible with the local scale symmetry discussed in Refs. [12,22], hence its name.

(2) The symmetry-breaking case $k=1$,

$$
\tau=\frac{2}{\sigma} \ln \frac{\mathrm{e}^{\sigma\left(T T_{d}\right) / 2}+\sqrt{\mathrm{e}^{\sigma\left(T-T_{d}\right)}+5 / 4}}{\mathrm{e}^{\sigma\left(T_{i}-T_{d}\right) / 2}+\sqrt{\mathrm{e}^{\sigma\left(T_{i}-T_{d}\right)}+5 / 4}} .
$$

This case resembles, but differs from the corresponding symmetry breaking solution obtained in the logPoisson case. As an example, we plot both solutions in Fig. 3 and compare them with the log-Poisson case. The data of Ref. [19] are also shown. We see that although different, both solutions fit the data equally well and could be used to describe these data.

Summarizing, we have studied both She-Lévêque and Castaing models from the point of view of scale invariance, and found no striking differences in their properties. Both account equally well for the scaling exponents, both explain the property of general scaling observed in turbulence and both give similar results when using a linear approximation. In fact, at the present stage of knowledge, they only differ by the regularity of the intermittency function: the moments for the thermodynamical model may divergence but the moments for the log-Poisson model always convergence. At the moment, we do not have any theoretical arguments ruling out the presence of divergencies in the scaling exponents. It is therefore only an act of faith to privilege one or the other model in the description of turbulent flows.

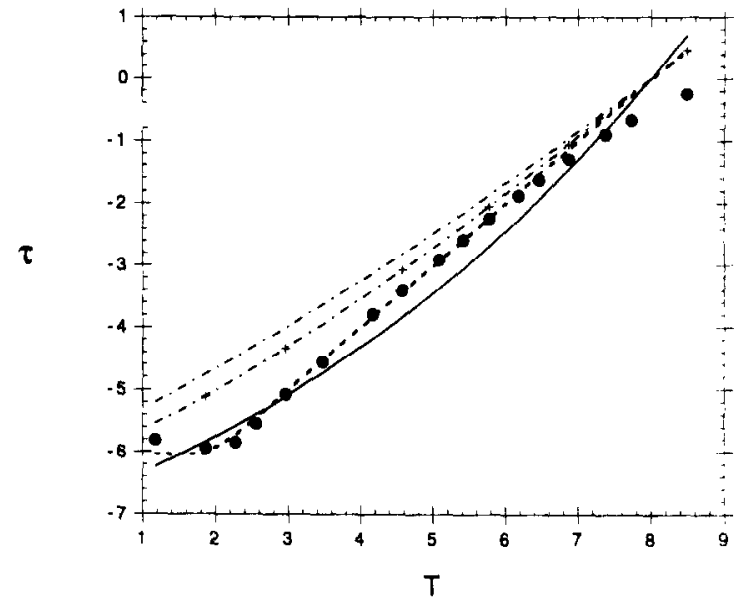

Fig. 3. The behavior of a the proper time in the obmic solution, shown with $T_{d}=2$ and $T_{i}=8$ as in the data of Ref. [19], shown with circles. In the symmetric case, this yields $\sigma=0.135$ in the $\log$-Poisson case and $\sigma / 2=0.135$ in the degenerate case so that both solutions coincide (full line). In the nonsymmetric case, $\sigma$ is a free parameter; high values, e.g. $\sigma=20$ for log-Poisson or $\sigma / 2=20$ coincide (dotted line) and provide the best fit, in contrast with low values, e.g. $\sigma=0.2$ (dashed-dotted line) for log-Poisson and $\sigma / 2=0.2$ (dashed-dotted line with crosses) for degenerate.

Thanks are due to to P. Tabeling and B. Castaing for their support and to SAP for its hospitality to G.W. He.

\section{References}

[1] A. Arnéodo et al., Europhys. Lett. 34 (1996) 411.

[2] A.N. Kolmogorov, Doklady Akad. Nauk. SSSR. 30 (1941) 301.

[3] U. Frisch, Turbulence: The legacy of A.N. Kolmogorov (Cambridge Univ. Press, Cambridge, 1995).

[4] Z.-S. She, E. Lévêque, Phys. Rev. Lett. 72 (1994) 336.

[5] B. Dubrulle, Phys. Rev. Lett 73 (1994) 959.

[6] Z.-S. She, E.C. Waymire, Phys. Rev. Lett. 75274 (1995).

[7] B. Castaing, J. Phys. II France 6 (1996) 105

[8] M. Nelkin, Phys. Rev. E 52 (1995) R4610.

[9] B. Chabaud, A. Naert, J. Peinke, F. Chilla. B. Castaing, B. Hébral, Phys. Rev. Lett. 73 (1994) 3227.

[10] B. Dubrulle, F. Graner, J. Phys. II France 6 (1996) 817.

[11] L. Nottale, Int. J. Mod. Phys. A 7 (1993) 4899; Fractal Space-Time and Microphysics (World Scientific, Singapore, 1993).

[12] A. Pocheau, Phys. Rev. E 49 (1994) 1109; Europhys. Lett. 35 (1996) 183.

[13] B. Dubrulle, F. Graner, J. Phys. II France 6 (1996) 797.

[14] F. Graner, B. Dubrulle, Phys Rev. E 56 (1997) 6427-6434.

[15] B. Dubrulle, F. Graner, Phys Rev. E 56 (1997) 6435-6442.

[16] E. Lévêque, Z.-S. She, Phys. Rev. Lett. 75 (1995) 2690. 
[17] R. Benzi et al., Physica D 96 (1996) 162.

[18] A. Vincent, M. Meneguzzi, J. Fluid Mech. 225 (1991) 1.

[19] F. Belin, P. Tabeling, H. Willaime, Physica D 93 (1996) 52.

[20] R. Camussi, G. Guj, J. Fluid Mech. 348 (1997) 177-199.

[21] H. Politano, A. Pouquet, P.L. Sulem, Phys. Plasmas 2 (1995) 2931.
[22] B. Dubrulle, J. Phys. II France 6 (1996) 1825.

[23] G. He, B. DubrulJe, J. Phys. Il France 7 (1997) 793-800.

[24] B. Castaing, Y. Gagne, M. Marchand, Physica D 68 (1993) 387.

[25] B. Castaing, J. Phys. II France 50 (1989) 147. 